\title{
Djerba, tourisme international et nouvelles logiques migratoires
}

\section{Élise Bernard}

\section{(2) OpenEdition \\ 1 Journals}

Édition électronique

URL : https://journals.openedition.org/remi/2629

DOI : $10.4000 /$ remi.2629

ISSN : $1777-5418$

Éditeur

Université de Poitiers

\section{Édition imprimée}

Date de publication : 1 mars 2002

Pagination : 103-112

ISBN : 2-911627-30-X

ISSN : 0765-0752

\section{Référence électronique}

Élise Bernard, «Djerba, tourisme international et nouvelles logiques migratoires », Revue européenne des migrations internationales [En ligne], vol. $18-n^{\circ} 1$ | 2002, mis en ligne le 09 juin 2006, consulté le 14 avril 2022. URL : http://journals.openedition.org/remi/2629; DOI : https://doi.org/10.4000/remi.2629

Ce document a été généré automatiquement le 14 avril 2022

(C) Université de Poitiers 


\title{
Djerba, tourisme international et nouvelles logiques migratoires
}

\author{
Élise Bernard
}

1 Djerba se situe dans le Sud tunisien, dans le golfe de Gabès. D’une superficie de 514 km², elle est densément peuplée (environ 150000 personnes en 2000). Pendant longtemps, l'île a été à l'origine d'un mode migratoire régional spécifique s'inscrivant dans le fait migratoire des régions périphériques de Tunisie. Ce rythme a été brisé à la fin des années soixante lorsque l'État tunisien a décidé d'y implanter un pôle touristique pour rompre avec les difficultés économiques de l'île. Ce projet s'inscrivait dans une politique plus large, à l'échelle tunisienne mais aussi à l'échelle des pays en voie de développement, misant sur le tourisme international pour développer l'économie et s'installer dans un réseau médiatique mondial. Outre son insularité, image touristique attrayante, l'île offrait des conditions de séjour agréables : mer chaude, climat modéré par rapport au Sud tunisien et paysages singuliers d' " oasis maritime ». L'installation du pôle touristique a eu des conséquences importantes sur les flux et les pratiques migratoires de l'île. Tout en engendrant, en provenance du continent, des flux migratoires internes sans cesse croissants, l'essor de cette nouvelle activité n'a pas mis fin à l'émigration internationale, contrairement à ce que pouvaient croire les autorités politiques. C'est cet antagonisme entre création d'emploi et émigration internationale qui a décidé de notre intérêt pour l'île de Djerba. Nous voulions comprendre comment les deux phénomènes pouvaient êtres concomitants et il nous paraissait particulièrement intéressant d'analyser la question des conséquences du tourisme dans un espace migratoire aussi structuré.

Le système djerbien traditionnel

2 Pendant des siècles, l'organisation de l'espace insulaire et la solide structuration de la société ont permis aux Djerbiens de quitter l'île sans craindre pour leur famille et leur patrimoine.

Une société insulaire solidement structurée

3 Le paysage de Djerba est la résultante d'une organisation lente et méthodique de l'espace par l'homme, traduisant un mode de vie et des relations sociales tout à fait 
particulières. Les Djerbiens ayant eu à subir des attaques répétées venant de la mer se sont éloignés de la côte et dispersés dans la campagne. L'île couverte d'édifices religieux révèle un espace insulaire profondément marqué par le sacré et une population profondément attachée à son territoire. le bâti est isolé et clairsemé à l'exception de trois groupements d'habitat. À cette dispersion de l'habitat se superpose une organisation hiérarchique de l'espace insulaire en niveaux emboîtés, à partir du menzel qui constitue la cellule de base de l'organisation spatiale de l'île. Les menzels sont des espaces résidentiels et fonctionnels dans lesquels vivent et s'organisent les familles. Le menzel, terme qui signifie la maison, désigne à Djerba l'aire de vie d'une famille. Il est formé d'un ou de plusieurs houch (unité d'habitation) et des éléments de la vie économique : vergers, champs mais aussi atelier de tissage, greniers, puits, et la citerne, indispensable à la vie familiale. Le menzel intègre sa population avec son milieu agricole. Entouré de tabias (haies de terre hérissée d'agaves, d'aloès et de figuiers de barbarie, bordant les pistes et les chemins et entourant les propriétés), il est organisé comme un site défensif.

4 Le cadre de la vie communautaire est la houma. Pour Hassan Boubakri (1985) « houma est un terme ethno-spatial désignant une sorte de terroir composé d'habitations, de parcelles cultivées, de puits et d'un espace social ", la mosquée étant l'élément fédérateur de l'ensemble. Dans chaque houma les traditions diffèrent. Les familles vivent en vase clos dans leur houma, sorte d'île dans l'île.

5 L'emboîtement en plusieurs niveaux est bien illustrée par l'organisation d'un réseau dense de sentiers hiérarchisés : pistes principales servant aux grandes distances, pistes secondaires desservant les houmas et en reliant certaines d'entre elles ensembles, passages desservant un groupement de menzel, passages desservant chacun uniquement un menzel.

6 Grâce à cette organisation précise de son territoire, le Djerbien, dont les ressources agricoles sont très insuffisantes par rapport aux besoins, part en migration en toute sécurité. La famille est organisée de telle sorte que l'absence de l'homme (l'émigration étant essentiellement masculine) ne mette pas en danger le déroulement de la vie du menzel. L'épouse travaille à proximité immédiate de la maison. , élève ses enfants, constamment sous la tutelle de sa belle-famille. Celle-ci est pour le migrant, un élément de confiance qui rend possible un long séjour à l'extérieur.

Une migration structurelle

7 La population nombreuse et l'insuffisance des ressources locales (le manque d'eau et de céréales, l'instabilité des récoltes, la concurrence dans le secteur de la pêche) sont à l'origine de crises conjoncturelles, liées le plus souvent lors de mauvaises récoltes. Elles ont contribué à la mise en place d'un processus migratoire important pour la vie de l'île, une émigration temporaire qui va petit à petit devenir structurelle en s'inscrivant dans le mode de vie permanent des Djerbiens, dans leur mode de relation à l'extérieur.

8 La grande majorité des Djerbiens en émigration pratiquent traditionnellement le commerce. La position stratégique de Djerba, à un carrefour du Sahara et de la Méditerranée, en fait, depuis longtemps un lieu de négoce. Selon Hassan Boubakri qui a étudié ce phénomène dans sa thèse Le petit commerce immigré du sud tunisien à Paris (1985), le commerce "est devenu au fil des années le secteur d'activité locale placé ailleurs, pour la population qui ne pouvait trouver des ressources sur place». Au XVIII ${ }^{\mathrm{e}}$ siècle les Djerbiens prennent la mer et s'installent en Turquie, en Libye et en Egypte où ils profitent des courants d'échanges maritimes et terrestres pour s'adonner au 
commerce. Au début du xxe siècle certains s'installent en Algérie, la plupart dans le département de Constantine. Mais la grande majorité reste en Tunisie, détenant le quasi monopole du commerce alimentaire et du commerce de détail. Le plus souvent le Djerbien tient une petite boutique de produits alimentaires et de produits manufacturés divers. Son personnel est presque toujours djerbien et recruté dans la famille.

Ce n'est qu'après l'Indépendance que la France devient la principale destination des migrants djerbiens (G.Simon 1979). Une des clefs de leur départ massif vers l'Europe à la fin des années cinquante est le plan de réformes entrepris par le ministre Ahmed Ben Salah et le président Habib Bourguiba où le commerce de détail est regroupé dans des coopératives, transformant les commerçants en salariés. Cette réforme n'a pas été du goût des Djerbiens qui perdaient ainsi leur indépendance et la possibilité d'avoir des revenus plus élevés que les salaires proposés par la coopérative. La grande majorité de ces commerçants s'installent dans l'agglomération parisienne, attirés par «la puissance économique et commerciale de la capitale française » (Boubakri, 1985). Le fonctionnement de la filière migratoire renforce cette tendance au regroupement.

La mise en place d'un réseau migratoire

10 Pour pouvoir revenir régulièrement dans l'île sans fermer leur commerce à l'extérieur, les Djerbiens est organisé en système de rotations fondé sur l'association, le plus souvent de trois personnes (le patron, l'employé et l'apprenti). Chacun à son tour prendra ses vacances à Djerba en s'occupant des intérêts de ses associés. Les migrants pratiquent ainsi un "va-et-vient » entre leur pays d'origine et leur lieu d'accueil.

11 Tout un réseau migratoire s'est ainsi mis en place permettant notamment au migrant de trouver un travail et au futur migrant de partir en migration. Une structure communautaire s'est crée dans le pays d'accueil, elle est à la fois agent de solidarité et de contrôle social. Elle garantit l'accueil et l'aide au nouvel arrivant et son encadrement dans la recherche d'un emploi. En effet, la filière commerçante donne la possibilité à tout membre du groupe ethnique d'apprendre le métier et de créer un commerce s'il en a les moyens, sinon d'être employé chez un commerçant de son groupe. Le métier est ici une véritable spécialité collective. Ainsi, le commerce ethnique en créant une offre favorise la circulation migratoire des entrepreneurs migrants par le recrutement de nouveaux employés ou associés dans le pays d'origine et le développement des activités sur les deux rives de la Méditerranée. Les travaux d'E. Ma Mung (1996) ont bien mis en évidence les liens entre entreprenariat ethnique, réseau familial et circulation migratoire. Ces liens sont particulièrement importants dans la migration djerbienne. L'ensemble de ces structures préexistait à la migration en France et elles ont permis une organisation précise de l'espace relationnel entre la France et Djerba. Les Djerbiens migrants ou anciens migrants rencontrés insistent beaucoup sur le réseau et la solidarité communautaire garants de leur pratique migratoire. Pour eux il est impensable de partir "à l'aventure » sans savoir où loger et sans avoir des perspectives de travail. Ils mettent aussi en avant le fait que leur migration est une migration "structurée, raisonnée, de valeur ».

Depuis deux décennies la pratique de l'espace transnational s'est densifiée. Avant les années quatre-vingts les migrants ne rentraient qu'une à deux fois par an et le retour, au moment de la retraite, était définitif. Aujourd'hui la fréquence ou la durée des retours est un signe de réussite, le fait de ne revenir qu'une fois par an est perçu négativement, mal supporté notamment par les épouses restées dans l'île. D’autre part, 
les mesures de restriction de l'immigration de travail en France de 1974 ont entrainé, à Djerba comme ailleurs, un important regroupement familial ; les familles qui vivent en France essayent pour la grande majorité d'entre elles de maintenir les liens avec le lieu d'origine, renforçant la circulation. Enfin, la plupart des migrants à la retraite pratiquent le va et vient, passant entre trois et six mois de l'année en émigration, ce qui leur permet de toucher leur retraite ou d'autres indemnités en France et de disposer ainsi d'un compte en devise à l'étranger et de la couverture sociale française.

La densité de la circulation migratoire s'est donc accrue. Les migrants qui pratiquent le plus souvent la navette entre espace d'origine et espace d'accueil sont ceux qui ont des affaires dans l'île. Ils sont ainsi nombreux à investir dans le secteur touristique.

Développement touristique et transformations de djerba

Le développement du tourisme à Djerba est le résultat d'une décision politique. L'Etat tunisien en a été l'acteur initial par ses investissements comme par les avantages fiscaux et financiers consentis aux établissements touristiques. Au départ, la politique hôtelière ne visait qu'une infrastructure simple et peu coûteuse, le maintien de l'activité touristique à un rang raisonnable. Ce n'est qu'en 1968, que Djerba est désignée comme zone prioritaire, secteur géographique à vocation touristique.

Développement d'un pôle touristique important pour la Tunisie

15 Après une période de relative stagnation dans les années soixante-dix, le tourisme a véritablement pris son essor dans les années quatre-vingts et quatre-vingt dix. On constate une véritable explosion des constructions nouvelles ainsi que une augmentation impressionnante de la capacité d'hébergement tandis que le nombre de visiteurs ne cesse de croître. Les investissements ont connu un nouvel accroissement à partir de la fin de la guerre du Golfe et sont restés à un niveau soutenu jusqu'en 1997. Depuis ils sont moins importants car de moins en moins de projets sont en cours et le secteur arrive à saturation. L'investissement dans la zone Djerba-Zarzis est passé de 100600 milliers de dinars ${ }^{1}$ en 1997 à 54225 milliers en 1999.

16 L'île de Djerba possède, en 2000, 98 hôtels avec une capacité de 34342 lits et cinq unités hôtelières sont en construction. Les hôtels de bon niveau (trois et quatre étoiles) possèdent les plus importantes capacités. En 1999 le taux d'occupation moyen de la capacité hôtelière à Djerba a été de $68 \%$, ce taux la situant en seconde position parmi les sites touristiques tunisiens. Le taux d'occupation annuel moyen est décroissant des hôtels les mieux classés aux hôtels non classés. La raison de ce phénomène s'explique par le contraste entre la régularité de la clientèle riche et la fluctuation de la clientèle de masse, fluctuante selon la période de l'année et les choix des voyagistes.

17 Un grand nombre de lits ne sont utilisés que l'été. De plus, le parc hôtelier vieillit vite, apparaît désuet, inconfortable et démodé, ce qui entraîne un tassement de la clientèle une réduction de la rentabilité. La concurrence impose des prix trop bas pour leur permettre une bonne maintenance, ce qui pose des problèmes de reconversion, car la durée de vie d'un produit hôtelier est limité, et au delà, il faut réadapter ou dévaluer. Or, la politique actuelle est plutôt à la course aux constructions qu'à la réadaptation des produits.

18 L'inadéquation est donc assez forte entre les projets des investisseurs et la réalité de la fréquentation. L'Etat a planifié le nombre de lits à obtenir mais a omis de planifier les démarches nécessaires pour attirer la clientèle et la faire rester. Pour maintenir et développer l'activité touristique, les principaux acteurs locaux sont favorables aujourd'hui à un enrichissement de l'offre touristique, à l'émergence de pôles 
d'attraction et d'activités nouvelles. Il y a donc contradiction entre le projet de l'Etat, un tourisme de masse, et les politiques locales qui souhaitent s'orienter désormais vers un tourisme de qualité aux retombées financières plus élevées. C'est à cette aspiration que répondent la création récente d'un terrain de golf et d'un casino comme la multiplication dans les hôtels d'installations de thalassothérapie en direction de la clientèle l'hiver.

19 Aujourd'hui la plupart des investissements touristiques dans l'île sont d'origine étrangère. Néanmoins les Djerbiens, qui affichaient volontiers un discours très réservé sur le tourisme, possèderaient en réalité, un tiers des hôtels de l'île (Miossec 1996).

Tourisme et transformations de l'économie et de l'espace djerbiens

La naissance et le développement d'un secteur touristique moderne sur le littoral oriental de l'île de Djerba ont été à l'origine de bouleversements profonds de la société, de l'économie, de l'espace.

21 L'urbanisation touristique a débouché sur le développement d'équipements lourds, nécessaires pour accueillir de gros contingents de touristes. Rapidement les infrastructures nécessaires furent mises en place. L'île qui était enclavée est désormais bien reliée au monde extérieur. Tout d'abord par l'intermédiaire de l'aéroport international de Djerba-Mellita dont la capacité ne cesse de croître : cette plate-forme a accueilli 1500000 de passagers en l'an 2000, la grande majorité des touristes arrivant par la voie des airs. De plus, la route d'el Kantara ainsi que des bacs modernes dont le nombre ne cesse d'augmenter, relient Djerba au continent. Le réseau routier se densifie. Avant 1960, l'île ne possédait qu'une soixantaine de kilomètres goudronnés. Aujourd'hui, non seulement les axes principaux, mais aussi les voies secondaires sont goudronnées et l'ensemble de l'île est bien desservi.

D'autre part, le réseau de télécommunications devient plus important, permettant à Djerba d'être mieux reliée au continent et à l'étranger. Familles et migrants peuvent ainsi plus facilement garder contact. De plus, avec l'apparition des hôtels les autorités se sont trouvées dans l'obligation d'électrifier Djerba et de l'alimenter en eau.

23 Le secteur touristique a aussi bouleversé l'économie locale en créant des emplois. Le nombre d'emplois directs, lié à l'hôtellerie, correspond à peu près au tiers du nombre de lits, ce qui correspond pour l'ensemble de la zone Djerba-Zarzis à quelques 15000 postes de travail. Ce nombre a quadruplé en treize ans.

24 Ce nouveau marché du travail est précaire, instable. Un certain nombre de ces emplois sont saisonniers. En outre, dans les hôtels, les entrées et les sorties d'emploi en cours d'année sont nombreuses, tout d'abord parce que le personnel peut très vite être limogé en fonction directe du marché de l'emploi, mais aussi parce que le personnel fluctue aussi de son plein gré, cherchant les meilleures places, les mieux payées.

Les emplois liés indirectement à la construction des complexes hôteliers (transports, services) sont encore plus nombreux, quelques 55 000. L'emploi indirect se développe encore plus rapidement que l'emploi direct. De nouvelles propositions de services fleurissent chaque jour suite à la diversification de la demande touristique.

Les implications migratoires du développement touristiqueUne immigration massive Dans l'économie traditionnelle de Djerba, l'immigration existait, mais elle restait temporaire et conjoncturelle sauf pour quelques rares groupes implantés. En effet, l'offre d'emplois saisonniers dans l'agriculture, attirait une main-d'œuvre venue des régions intérieures du Sud. Les effets de l'émigration des Djerbiens en France et ceux du 
tourisme l'ont transformée. L'immigration qui était saisonnière et masculine devient familiale et définitive. L'aire de recrutement des immigrants s'élargit, touche désormais l'ensemble de la Tunisie avec néanmoins une prédominance pour les régions des steppes et du Sud.

Pour les Tunisiens du continent, aller travailler à Djerba représente un substitut à la migration temporaire en France, exprimant comme le souligne R. Escallier (1995) «le rétrécissement des champs migratoires et la forte prépondérance des mouvements locaux». L'offre d'emplois touristiques coïncide en effet, avec la fermeture des frontières européennes et l'importance, dans toute la Tunisie, des départs des ruraux vers les villes et le littoral. La migration interne est, certes, générée par l'attirance que procure le mode de vie urbain mais aussi au fait que les ressources fournies par la terre sont désormais jugées insuffisantes face aux besoins croissants d'une société souhaitant accéder au statut de "société de consommation ». Cette immigration d'installation devient donc structurelle pour l'île de Djerba; elle représente environ $45 \%$ de l'ensemble des habitants de l'île et $60 \%$ des actifs ; les Djerbiens doivent composer avec cette nouvelle population.

L'immigration familiale est de deux types : la migration familiale élargie et la migration " pionnière ». La première est présente dans l'île sous forme de tribus, majoritairement du Sud, qui s'installent dans la campagne djerbienne pour mener des activités agricoles, travailler dans les carrières ou dans le bâtiment. Mais la migration familiale la plus pratiquée est la forme classique du mari qui, une fois qu'il a trouvé du travail, est rejoint par sa femme et ses enfants.

Les nouveaux arrivants viennent de toute la Tunisie. À la première vague d'arrivants qui étaient surtout ouvriers dans le bâtiment s'est substituée une population active beaucoup plus hétérogène. Les premiers occupaient des niches d'emplois délaissées ou jamais occupées par les Djerbiens, alors que les nouveaux arrivants concurrencent les Djerbiens dans leurs activités ou dans leur accès à l'emploi. En effet, si dans les premières années du développement du tourisme, cette activité a peu attiré les Djerbiens, aujourd'hui ceux-ci cherchent à accéder aux emplois disponibles.

30 L'origine des travailleurs dans le tourisme est diverse comme nous le montre l'analyse menée à partir des sources du complexe hôtelier Dar Djerba. Les Djerbiens représentent $48 \%$ des effectifs. Parmi les non Djerbiens, $35 \%$ sont originaires du gouvernorat de Médenine, $13 \%$ de celui de Tunis, $9 \%$ de celui de Gafsa, enfin ceux de Gabès et Tataouine ont fourni chacun $6 \%$ des nouveaux arrivants. Outre Tunis, ce sont donc surtout les gouvernorats du Sud qui fournissent le gros des effectifs. En comparant les chiffres pour ce même complexe de 1976 et de 2001 on remarque que les employés originaires du Sud sont mieux représentés aujourd'hui. Les réticences face à l'emploi touristique ont disparu et la main-d'œuvre qualifiée dans l'hôtellerie qui en 1976 était originaire du Nord, est désormais formée dans les écoles hôtelières d'Houmt-Souk (principal centre urbain de l'île) et de Tozeur.

31 La majorité des personnes travaillant dans l'hôtellerie appartiennent à la tranche d'âge des 26-45 ans, avec un niveau de formation secondaire, ce qui traduit une élévation sensible du niveau de qualification par rapport au démarrage de ce complexe hôtelier. Depuis le personnel touristique est de plus en plus qualifié. Les 15-25 ans représentent la grosse masse des travailleurs saisonniers. Ces quelques données expriment bien les tendances actuelles de l'hôtellerie. Les hôtels embauchent peu de salariés permanents pour minimiser les coûts en raison de la concurrence féroce entre les établissements et 
des fortes fluctuations de l'activité. Ce mode de gestion réduit ainsi les jeunes à une constante instabilité et à un sous-emploi.

Une nouvelle émigration internationale djerbienne

Les formes d'emploi, qui ne leur permettent pas d'être rassurés sur leur avenir et de former des projets, pousse les jeunes à choisir comme ultime solution l'émigration internationale. L'île de Djerba sert donc de tremplin vers l'étranger car elle leur permet de nouer des contacts avec les touristes dans l'espoir de partir à l'étranger grâce à une lettre d'accueil et, dans certains cas, un mariage. Le tourisme est "directement responsable d'une nouvelle forme d'émigration depuis que les mesures législatives mises en place par les pays européens ont fortement réduit l'émigration légale : les mariages mixtes " (Kassah, 1997).

Les jeunes Tunisiens peuvent difficilement choisir et planifier leur vie professionnelle; ils savent aussi qu'il leur sera difficile d'atteindre le niveau de vie occidental auquel ils aspirent. Ils en conçoivent de l'amertume et pour ne pas désespérer cherchent un ailleurs qui se trouvent dans l'émigration. Beaucoup de ceux que nous avons rencontrés ont une vision très pessimiste de l'avenir de leur pays. Ce désir est plus fort dans les classes instruites, qui souvent ont déjà un emploi leur permettant de satisfaire plus ou moins aisément les besoins essentiels. Le sentiment est qu'aujourd'hui les rêves des jeunes maghrébins sont plus ou moins les mêmes que ceux des jeunes occidentaux, c'est-à-dire, ceux d'une société de consommation riche. Ils ont également l'impression que tout se passe ailleurs. L'étranger rime pour eux avec vie facile, gains faciles, libertés, avenir. Beaucoup aspirent à une vie sociale moins contraignante et à une vie culturelle plus libre. Ils sont d'autant plus sensibles aux modes de vie occidentaux qu'ils sont jeunes et déjà prédisposés à tenter leur chance à l'extérieur par l'enseignement et par la formation qu'ils ont reçue.

De plus, les jeunes qui travaillent dans le tourisme se lassent assez vite de leur travail et comme il est difficile de se reconvertir, car l'offre d'emplois manque, ils décident donc de partir à l'étranger d'autant plus facilement qu'ils ont l'illusion de connaitre ces étrangers qui viennent chez eux. Le tourisme met l'Occident sous le regard permanent : le rêve de la migration est toujours présent, voire même amplifié.

Dans ce contexte, l'émigration djerbienne est en train de perdre ses spécificités. Elle fonctionne de moins en moins en réseaux, sauf dans le cas de la migration clandestine, mais elle devient le fait de pratiques européennes. L'extrême polarisation vers Paris des années soixante-dix et quatre-vingt s'affaiblit;la migration se diffuse. En effet, les Djerbiens suivent leurs compagnes et se dispersent en France, en Allemagne et dans une moindre proportion en Suisse et en Italie. On assiste à une certaine perte de la spécialité commerçante qui nécessitait une grande solidarité et cohésion du groupe, les nouveaux migrants n'ont plus d'activité cible. Le choix de ce type de migration induit le désir d'un changement dans le mode d'existence. Les migrants qui prennent ces risques veulent pour la plupart accéder au mode de vie européen. Le seul acte du mariage mixte entraîne directement le migrant dans un processus de mélange des cultures voir d'acculturation.

Perdant son caractère temporaire, l'émigration tend à devenir définitive pour les jeunes migrants, mais aussi pour bon nombre de familles désormais installées en France. On peut se demander quel est le devenir de l'espace relationnel entre Djerba et l'Europe. Quel peut être l'impact socio-économique pour l'île de Djerba d'une migration définitive, à part l'aide plus ou moins régulière aux familles restées sur place et 
éventuellement la construction d'une villa secondaire pour les vacances? La migration internationale est en train de perdre son rôle structurel dans l'économie insulaire où elle est supplantée par le tourisme.

Le tourisme a, certes, contribué au désenclavement de Djerba en la dotant d'un certain nombre d'infrastructures modernes mais le développement exclusif de la fonction touristique comporte des risques car il se fait aux dépens d'autres activités. Or, quelle que soit l'évolution du tourisme, la question de l'emploi de la population reste posée. Cette question est d'autant plus prégnante que le tourisme a entrâné une vague d'immigration interne importante lié à l'attrait d'un niveau de vie plus élevé. Si le développement du pôle touristique djerbien s'arrête, comment l'île fournira-t-elle fournir un emploi à tous les actifs installés dans l'île?

Une société locale déboussolée?

Depuis les années soixante la société djerbienne s'est transformée. Ces changements sont dus à la fois à l'émigration, à l'immigration et au tourisme international sans oublier les impacts du développement de la scolarité et celui de la télévision. L'ensemble de ces facteurs s'enchevêtre et il est difficile de distinguer le rôle de chacun. Néanmoins ils ont tous mené à la déstructuration d'un mode de vie insulaire spécifique et à l'affaiblissement de la houma. Les jeunes se sentent de moins en moins appartenir à une communauté. Les hommes et les femmes qui souhaitent accéder au mode de vie occidental rejettent l'institution du mariage traditionnel et voient la solution à leur quête dans le mariage mixte. L'écart entre les deux cultures et l'écart entre leurs envies et la possibilité de les satisfaire engendre chez les jeunes de vives réactions et parfois des comportements déviants. Le développement de nouveaux besoins ne pouvant être satisfaits faute d'emplois suffisants et bien rémunérés engendre une émigration à la recherche d'un nouveau mode de vie. Elle reste perçue comme le plus sûr moyen d'ascension sociale. On aurait pu penser que les emplois touristiques signifieraient la fin de l'émigration djerbienne, il n'en est rien. A Djerba, le tourisme a bouleversé la logique de la migration internationale ; il ne l'a pas supprimée.

\section{BIBLIOGRAPHIE}

BERNARD Élise (2001) Djerba : impact des mutations engendrées par l'implantation d'un pôle touristique sur une île tournée vers l'émigration internationale. Mém. maîtrise de géographie (direct. G. Simon). Université de Poitiers, $141 \mathrm{p}$.

BOUBAKRI Hassan (1985) Le petit commerce immigré du sud-Tunisien à Paris : Espace, fonctionnement social et impact sur les régions natales, Strasbourg : Université Louis Pasteur, Th. doct. : Géogr. : Strasbourg, 299 p.

ESCALLIER Robert (1995) Populations et systèmes migratoires du monde arabe, in Troin JeanFrançois (dir.), Maghreb, Moyen-Orient, mutations, Paris, SEDES, pp. 179-213.

GY-JAABIRI Delphine (1999) Migrations, tourisme et développement local à Djerba (Tunisie) Poitiers : université de Poitiers, UFR sciences humaines et arts, Mém. DEA. : Géogr : Poitiers : 2000, 108 p. 
KASSAH Abdefettah (1997) Les mouvements migratoires et leurs effets sur l'île de Jerba, in Berriane Mohamed, Popp Herbert, Migrations internationales entre le Maghreb et l'Europe : les effets sur les pays de destination et d'origine, Passau 1998 : Actes du colloque maroco-allemand de München 1997, pp. 181-186.

MA MUNG Emmanuel (dir.) (1996) Mobilités et investissements des émigrés. Maroc, Tunisie, Turquie, Sénégal, Paris, l'Harmattan, 272 p.

MIOSSEC Jean-Marie (1996) Le tourisme en Tunisie, un pays en développement dans l'espace touristique international, Thèse de Doctorat d'Etat, Université de Tours, 1333 p.

MZABI Hassouna (1992) La Tunisie du Sud-Est, géographie d'une région fragile, marginale et dépendante, Tunis : Faculté de Sciences Humaines et Sociales, série géographique, vol. 30, Th. Etat ès Sciences Humaines et sociales, Tunis, 1988, 685 p.

MZABI Hassouna (1976) L'emploi et les investissements touristiques à Djerba, Revue Tunisienne de Géographie, n², pp. 111-134.

SIMON Gildas (1979) L'espace des travailleurs tunisiens en France : structure et fonctionnement d'un champ migratoire international, Poitiers : Université de Poitiers, UFR Sciences humaines et arts, Th État : Géogr. : Poitiers : 1978, 426 p.

STABLO René (1940) Les Djerbiens, une communauté arabo-berbère dans une île de l'Afrique Française, Tunis, $164 \mathrm{p}$.

TLATLI Salah-Eddine (1967), L'île des Lotophages, Tunis, CERES Productions, 192 p.

\section{NOTES}

1. Un dinar = environ 0,76 euros.

\section{AUTEUR}

\section{ÉLISE BERNARD}

Étudiante en géographie, Université de Poitiers 